\title{
Role of the EGFR-KDD mutation as a possible mechanism of acquired resistance of non-small cell lung cancer to EGFR tyrosine kinase inhibitors: A case report
}

\author{
CHENG HE $^{1,2}$ and YONG WANG ${ }^{2}$ \\ ${ }^{1}$ Department of Thoracic Oncology, Anhui Provincial Cancer Hospital; ${ }^{2}$ Department of Medical Oncology, \\ The First Affiliated Hospital of University of Science and Technology of China, Hefei, Anhui 230000, P.R. China
}

Received January 1, 2021; Accepted August 4, 2021

DOI: $10.3892 / \mathrm{mco} .2021 .2463$

\begin{abstract}
Epidermal growth factor receptor-tyrosine kinase inhibitors (EGFR-TKIs) are currently considered as the standard therapy for patients with advanced non-small cell lung cancer (NSCLC) who have EGFR-activating mutations. However, despite an initially profound response to these drugs, these patients ultimately develop drug resistance. The most common resistance mechanism is the development of a secondary mutation in EGFR (T790M), although activation of the MNNG/HOS transforming gene (MET), amplification of the Erb-B2 receptor tyrosine kinase 2 gene and histological transformation to small cell lung cancer may also lead to resistance. In addition, there may be additional, rare mechanisms leading to resistance that remain unidentified. Mutations in the EGFR kinase domain duplication (EGFR-KDD) are rare, although they act as oncogenic drivers in NSCLC. To the best of our knowledge, all studies to date have reported EGFR-KDD as the primary mutation in NSCLC. The aim of the present study was to report the case of an EGFR-KDD mutation in a patient with NSCLC who developed acquired resistance to gefitinib, but responded well to afatinib. Therefore, EGFR-KDD mutation is an additional potential mechanism underlying the development of acquired resistance to EGFR-TKIs.
\end{abstract}

\section{Introduction}

Several oncogenic alterations, such as mutations in the epidermal growth factor receptor (EGFR) gene, occur in non-small cell lung cancer (NSCLC) (1). The results from

Correspondence to: Professor Yong Wang, Department of Medical Oncology, The First Affiliated Hospital of University of Science and Technology of China, 17 Lujiang Road, Hefei, Anhui 230000, P.R. China

E-mail: chwangyong@126.com

Key words: non-small cell lung cancer, epidermal growth factor receptor kinase domain duplication, EGFR tyrosine kinase inhibitor, acquired resistance several large phase III trials demonstrated that, relative to chemotherapy, EGFR tyrosine kinase inhibitors (EGFR-TKIs) significantly improved the survival rates of patients with NSCLC who had EGFR-activating mutations (2-4). Consequently, EGFR-TKIs, such as gefitinib (first-generation) and afatinib (second-generation), are now approved worldwide, and are currently used as first-line treatments for patients with NSCLC harboring EGFR mutations.

Although EGFR-TKIs initially achieve a notable response, almost all patients eventually acquire drug resistance. Previous studies have reported that the EGFR T790M mutation accounted for $50-60 \%$ of all cases of acquired resistance to EGFR-TKIs. Amplification of the MNNG/HOS transforming gene (MET), histological transformation to small cell lung cancer and mutation of the Kirsten rat sarcoma viral oncogene homolog may also lead to resistance $(1,5,6)$. The development and application of next-generation sequencing (NGS) technologies have enabled the identification of rare mutations. For example, Gallant et al (7) identified a mutation in the kinase domain duplication (KDD) of the EGFR gene (EGFR-KDD) acting as an oncogene in NSCLC.

We herein report a rare case of an EGFR-KDD mutation that conferred resistance to gefitinib in a patient with NSCLC, who subsequently responded well to afatinib treatment.

\section{Case report}

In September 2015, a 56-year-old male smoker who presented with complaints of mild hemoptysis for 2 months was admitted to The First Affiliated Hospital of the University of Science and Technology of China (Hefei, China). A chest CT scan revealed a lesion in the lower lobe of the right lung, and a positron emission tomography/CT scan showed a marked increase of fluorodeoxyglucose (FDG) uptake in this lesion. No FDG accumulation was identified in other parts of the body and, therefore, a right-lower lobectomy was performed. The postoperative histopathological examination indicated an invasive non-mucinous adenocarcinoma. According to the 8th edition of the American Joint Committee on Cancer TNM staging system for NSCLC (8), the cancer was classified as stage IIb (T1bN1M0). The patient subsequently received four cycles of gemcitabine $\left(1,000 \mathrm{mg} / \mathrm{m}^{2}\right.$ i.v. on $\mathrm{d} 1$ and $\left.\mathrm{d} 8\right)$ plus cisplatin 
A

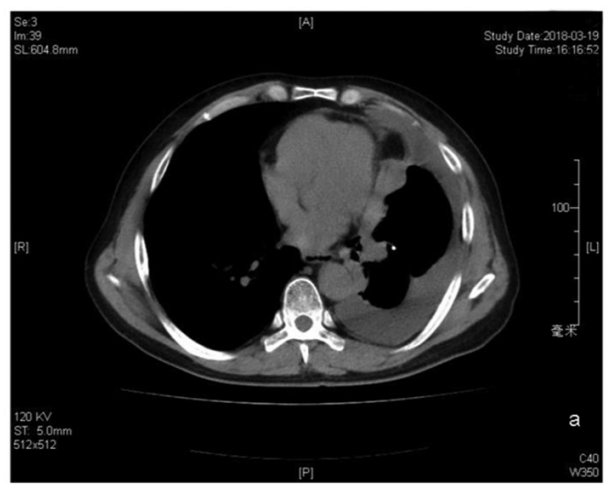

B

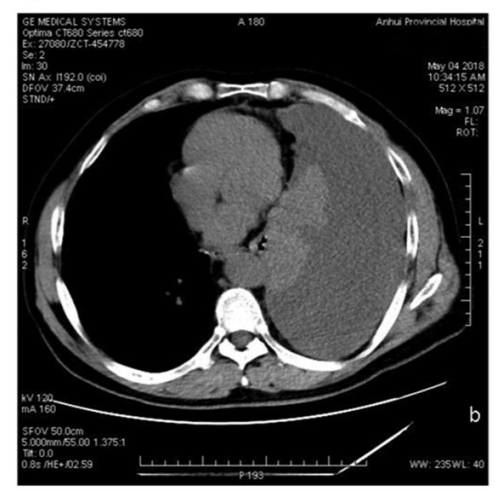

C

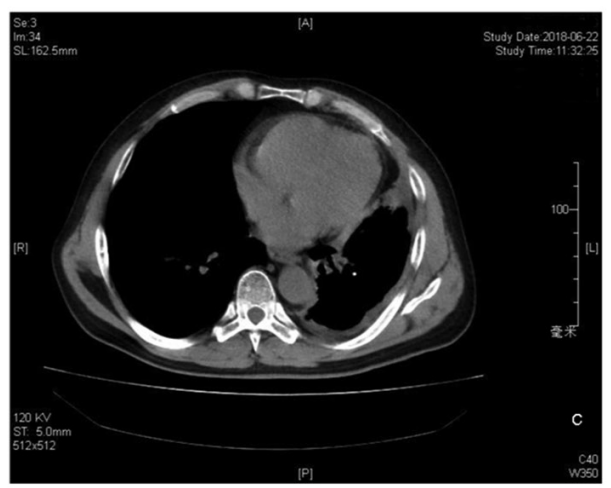

Figure 1. (A) Chest CT scan performed in March 2018, showing a right-sided pleural effusion and multiple pleural nodules. (B) CT scan performed in in May 2018, showing a massive right-sided pleural effusion. (C) Chest CT scan performed in in June 2018 (after afatinib treatment), showing no pleural effusion and a reduction in the size of the pleural nodules.

( $75 \mathrm{mg} / \mathrm{m}^{2}$ i.v. on $\mathrm{d} 1$ ) as postoperative adjuvant chemotherapy, and was followed up every 3 months thereafter.

In August 2016, a contrast-enhanced chest CT scan revealed mediastinal lymphadenopathy and multiple pleural nodules with heterogeneous enhancement, indicating recurrence of the lung cancer. A mutation in EGFR exon 21 (L858R) was detected in the patient's surgically resected tissue using the amplification-refractory mutation system (ARMS; Amoy Diagnostics Co., Ltd.). The patient was treated with gefitinib (250 mg p.o. qd) and achieved stable disease over the following 21 months.

In March 2018, the patient visited our hospital again for routine examination. A chest CT scan revealed right-sided pleural effusion and multiple pleural nodules scattered within the entire right pleura, which ranged in size from 5 to $15 \mathrm{~mm}$ (Fig. 1A). However, as the patient felt well, he refused to change the treatment regimen at that time.

After 2 months, the patient revisited our hospital complaining of dyspnea. Another CT scan revealed a massive right-sided pleural effusion (Fig. 1B), which necessitated thoracentesis using a central intrathoracic venous catheter to drain the malignant pleural fluid. Pleural fluid cytological examination revealed the presence of adenocarcinoma cells. Only a few adenocarcinoma cells were detected; therefore, genetic testing could not be performed. We therefore used NGS (HiSeq/MiSeqDx, Illumina, Inc.; performed by Geneseeq Technology Inc.) of a blood sample to detect the possible mechanism of resistance. The sequencing depth of the target area was 5,000x and the coverage of the target area was $99.8 \%$. The results revealed that the mutation in EGFR exon 21 (L858R) had disappeared, and a new EGFR-KDD mutation was identified (Fig. 2). Amplification of the cyclin-dependent kinase $(\mathrm{CDK}) 4$ gene and a mutation in the SMARCA4 gene, which encodes a protein in the SWitch/Sucrose Non-Fermentable family and functions in DNA remodeling, were also identified.

Therefore, afatinib treatment (40 mg p.o. qd) was initiated. One month later, a chest CT scan indicated no pleural effusion and a reduction in the size of the pleural nodules (Fig. 1C). At the 9-month follow-up, there was no evidence of recurrence. In March 2019, the patient returned to our hospital and reported experiencing a feeling of tiredness. A chest CT scan revealed a $\sim 2$-fold increase in the size of the pleural nodules, and NGS-based liquid biopsy of blood samples revealed that the EGFR-KDD mutation was still present. There was also a decreased abundance of EGFR-KDD and increased amplification of CDK4 (Table I). Therefore, treatment with palbociclib (125 mg once daily taken with food for 21 days followed by 7 days off treatment) was attempted; palbociclib is an oral pyridopyrimidine-derived CDK inhibitor that was previously proposed as a therapy for overcoming afatinib resistance in patients with NSCLC (9). However, 1 month later, another chest $\mathrm{CT}$ scan revealed a marked increase in the size of multiple tumor nodules. Two more cycles of pemetrexed (500 mg/m² i.v. on d1) plus bevacizumab (7.5 mg/ $\mathrm{kg}$ i.v. on d1) were administered; however, the patient succumbed to the disease in September 2019.

\section{Discussion}

A mutation in EGFR-KDD, which was first described as an oncogenic driver of NSCLC in 2015, consists of an in-tandem duplication of exons 18-25 (7). Functionally, the tandem connection of tyrosine kinase domains may form an intramolecular dimer that confers constitutive activation of EGFR (10). To date, there are only few case reports of this rare mutation in patients with NSCLC $(11,12)$. A recent multicenter study of 10,579 patients with NSCLC reported that only $0.12 \%$ of the cases harbored the EGFR-KDD mutation (13). Notably, all those studies described this as the primary mutation in NSCLC. To the best of our knowledge, there are no previous reports on the role of the EGFR-KDD mutation in acquired resistance to EGFR-TKIs.

In the present study, ARMS was used to detect the L858R point mutation in EGFR exon 21 in a patient with lung adenocarcinoma who developed postoperative recurrence. Gefitinib was initially administered; however, after disease progression, an NGS-based liquid biopsy was used to examine the possible mechanism underlying resistance development. Despite the disappearance of the EGFR exon 21 L858R mutation, a new EGFR-KDD mutation and CDK4 gene amplification were identified.

Previous studies have reported evidence that afatinib treatment achieved promising effects in patients with NSCLC 
Table I. Dynamic alteration of CNV or mutation during this case of adenocarcinoma.

\begin{tabular}{lccc}
\hline $\begin{array}{l}\text { CNV or } \\
\text { mutation }\end{array}$ & August 2016 & $\begin{array}{c}\text { Date } \\
\text { May 2018 }\end{array}$ & March 2019 \\
\hline Abundance of EGFR p.L858R mutation & $19.9 \%$ & - & - \\
Abundance of TP53 mutation & $20.4 \%$ & $5.1 \%$ & - \\
Abundance of EGFR-KDD mutation & - & $11.8 \%$ & $1.3 \%$ \\
Abundance of SMARCA4 mutation & - & - & $4.8 \%$ \\
Copy number of EGFR amplification & - & - & 5 \\
Copy number of CDK4 amplification & - & & 4 \\
\hline
\end{tabular}

CNV, copy number variation; EGFR, epidermal growth factor receptor; EGFR-KDD, EGFR kinase domain duplication; SMARCA4, SWitch/Sucrose Non-Fermentable family-related, matrix associated, actin-dependent regulator of chromatin, subfamily a, member 4; CDK4, cyclin-dependent kinase 4; -, undetected.

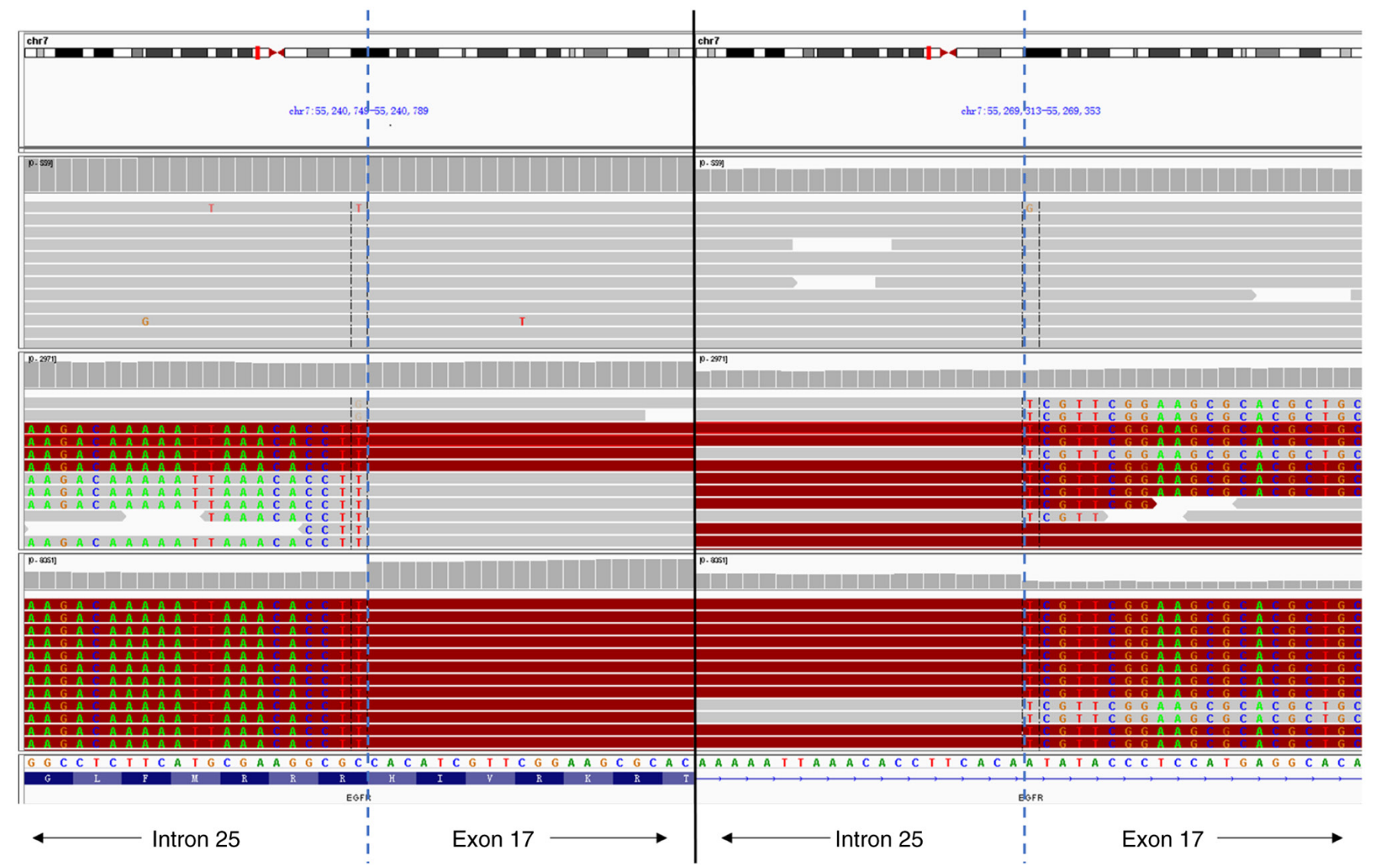

Figure 2. Integrative Genomics Viewer on the EGFR kinase domain duplication site of the blood cell negative control, plasma and tumor tissue samples indicating an in-tandem duplication of the complete kinase domain (EGFR exon 18 to exon 25). The dashed lines in blue indicate genomic breakpoints located on exon 17 and intron 25. The colored letters indicate the mismatched bases around the breakpoint.

who harbored uncommon EGFR mutations, such as those with EGFR-KDD $(11,13,14)$. In addition, the results from our previous clinical practice indicated that several patients with NSCLC who had the EGFR G719X mutation and acquired resistance to gefitinib, nonetheless responded well to afatinib. Therefore, the patient in the present case was treated with afatinib. After 1 month, the patient reported no chest tightness, and the pleural effusion had disappeared. This was accompanied by prolonged stable disease for 10 months.
To ascertain whether the EGFR-KDD mutation identified was a primary mutation, NGS on surgically resected tissue was performed. There was no evidence of other mutations, except those in EGFR exon 21 (L858R) and TP53.

SMARCA4 alterations are the most common recurrent genomic alterations in NSCLC, and they have been found to be associated with poor patient outcome (15). However, the present case exhibited a decreased abundance of the SMARCA4 mutation following EGFR-TKI treatment. This 
result suggested that a SMARCA4 mutation did not play a central role in the progression of NSCLC in our patient.

When the patient's condition worsened again, a decreased abundance of the EGFR-KDD mutation and an increase in CDK4 amplification were identified. Consequently, it was hypothesized that the EGFR-KDD mutation may have conferred resistance to gefitinib, subsequently becoming the new driver mutation, instead of the initial EGFR L858R mutation.

Various mechanisms of acquired resistance to first-generation EGFR-TKIs in patients with NSCLC who had EGFR mutations have been previously described, and the T790M mutation is the most common known mechanism underlying resistance (16). Chemotherapy remains the standard therapy for patients with T790M-negative NSCLC who have acquired resistance to EGFR-TKIs. However, the patient described herein had an acquired EGFR-KDD mutation, and yet responded well to afatinib following failure of gefitinib. This rare mutation was detected by liquid biopsy using an NGS assay, which is a non-invasive technology that can provide dynamic monitoring of gene mutations for targeted therapy of patients with NSCLC (17). NGS is not widely available in China due to the high cost. However, the PIONEER study showed that over half of Asian patients with NSCLC harbor EGFR mutations (18). Therefore, more widespread use of NGS should enable the detection of more rare acquired mutations, thereby identifying new targets for the development of novel therapies.

In conclusion, the EGFR-KDD mutation in NSCLC may be a secondary EGFR mutation that confers resistance to treatment with first-generation EGFR-TKIs, such as gefitinib, but appears to be sensitive to the second-generation EGFR-TKI, afatanib. Identification of this mutation in additional patients with NSCLC will confirm its role in acquired resistance.

\section{Acknowledgements}

Not applicable.

\section{Funding}

No funding was received.

\section{Availability of data and materials}

The datasets generated and analyzed during the current study are not publicly available, as it is not allowed to share the sequencing data of Chinese patient without the permission according to the Criminal Law of The People's Republic of China, but are available from the corresponding author on reasonable request.

\section{Authors' contributions}

$\mathrm{CH}$ : Writing the original draft of the manuscript; YW: Conceptualization, supervision, writing, review and editing of the manuscript. Both authors have seen and can confirm the authenticity of the raw data. Both authors have read and approved the final version of the manuscript.

\section{Ethics approval and consent to participate}

Not applicable.

\section{Patient consent for publication}

The patient provided written consent for the publication of the case details and any accompanying images.

\section{Competing interests}

The authors declare that they have no competing interests.

\section{References}

1. Lim ZF and Ma PC: Emerging insights of tumor heterogeneity and drug resistance mechanisms in lung cancer targeted therapy. J Hematol Oncol 12: 134, 2019.

2. Mok TS, Wu YL, Thongprasert S, Yang CH, Chu DT, Saijo N, Sunpaweravong P, Han B, Margono B, Ichinose Y, et al: Gefitinib or carboplatin-paclitaxel in pulmonary adenocarcinoma. N Engl J Med 361: 947-957, 2009.

3. Lee SM, Khan I, Upadhyay S, Lewanski C, Falk S, Skailes G, Marshall E, Woll PJ, Hatton M, Lal R, et al: First-line erlotinib in patients with advanced non-small-cell lung cancer unsuitable for chemotherapy (TOPICAL): A double-blind, placebo-controlled, phase 3 trial. Lancet Oncol 13: 1161-1170, 2012.

4. Rosell R, Carcereny E, Gervais R, Vergnenegre A, Massuti B, Felip E, Palmero R, Garcia-Gomez R, Pallares C, Sanchez JM, et al: Erlotinib versus standard chemotherapy as first-line treatment for European patients with advanced EGFR mutation-positive non-small-cell lung cancer (EURTAC): A multicentre, open-label, randomised phase 3 trial. Lancet Oncol 13: 239-246, 2012.

5. Ko B, Paucar D and Halmos B: EGFR T790M: Revealing the secrets of a gatekeeper. Lung Cancer (Auckl) 8: 147-159, 2017.

6. Normanno N, Maiello MR, Chicchinelli N, Iannaccone A, Esposito C, De Cecio R, D'alessio A and De Luca A: Targeting the EGFR T790M mutation in non-small-cell lung cancer. Expert Opin Ther Targets 21: 159-165, 2017.

7. Gallant JN, Sheehan JH, Shaver TM, Bailey M, Lipson D, Chandramohan R, Red Brewer M, York SJ, Kris MG, Pietenpol JA, et al: EGFR kinase domain duplication (EGFR-KDD) is a novel oncogenic driver in lung cancer that is clinically responsive to afatinib. Cancer Discov 5: 1155-1163, 2015.

8. Lababede $\mathrm{O}$ and Meziane MA: The eighth edition of TNM staging of lung cancer: Reference chart and diagrams. Oncologist 23: 844-848, 2018

9. Nie H, Zhou X, Shuzhang D, Nie C, Zhang X and Huang J: Palbociclib overcomes afatinib resistance in non-small cell lung cancer. Biomed Pharmacother 109: 1750-1757, 2019.

10. Du Z, Gallant JN, Sheehan J, Meiler J and Lovly CM: Intramolecular dimerization of EGFR kinase domain duplication as a novel activation mechanism. J Thoracic Oncol 12 (Suppl): S1536, 2017.

11. Baik CS, Wu D, Smith C, Martins RG and Pritchard CC: Durable response to tyrosine kinase inhibitor therapy in a lung cancer patient harboring epidermal growth factor receptor tandem kinase domain duplication. J Thoracic Oncol 10: e97-e99, 2015.

12. Zhu YC, Wang WX, Xu CW, Tan QH, Li JY, Zhuang W, Song ZB, Du KQ, Chen G, Lv TF and Song Y: Lung adenocarcinoma patient with an EGFR kinase domain duplication (KDD) and the response to icotinib. J Thorac Dis 10: E359-E363, 2018.

13. Wang J, Li X, Xue X, Ou Q, Wu X, Liang Y, Wang X, You M, Shao YW, Zhang Z and Zhang S: Clinical outcomes of EGFR kinase domain duplication to targeted therapies in NSCLC. Int J Cancer 144: 2677-2682, 2019.

14. Russo A, Franchina T, Ricciardi G, Battaglia A, Picciotto M and Adamo V: Heterogeneous responses to epidermal growth factor receptor (EGFR) tyrosine kinase inhibitors (TKIs) in patients with uncommon EGFR mutations: New insights and future perspectives in this complex clinical scenario. Int J Mol Sci 20: 1431, 2019. 
15. Schoenfeld AJ, Bandlamudi C, Lavery JA, Montecalvo J, Namakydoust A, Rizvi H, Egger J, Concepcion CP, Paul S, Arcila ME, et al: The genomic landscape of SMARCA4 alterations and associations with outcomes in patients with lung cancer. Clin Cancer Res 26: 5701-5708, 2020.

16. Westover D, Zugazagoitia J, Cho BC, Lovly CM and Paz-Ares L: Mechanisms of acquired resistance to first- and second-generation EGFR tyrosine kinase inhibitors. Ann Oncol 29 (Suppl 1): i10-i19, 2018.

17. Rolfo C, Mack PC, Scagliotti GV, Baas P, Barlesi F, Bivona TG, Herbst RS, Mok TS, Peled N, Pirker R, et al: Liquid biopsy for advanced non-small cell lung cancer (NSCLC): A statement paper from the IASLC. J Thorac Oncol 13: 1248-1268, 2018.
18. Shi Y, Au JS, Thongprasert S, Srinivasan S, Tsai CM, Khoa MT, Heeroma K, Itoh Y, Cornelio G and Yang PC: A prospective, molecular epidemiology study of EGFR mutations in Asian patients with advanced non-small-cell lung cancer of adenocarcinoma histology (PIONEER). J Thorac Oncol 9: 154-162, 2014.

This work is licensed under a Creative Commons Attribution-NonCommercial-NoDerivatives 4.0 International (CC BY-NC-ND 4.0) License. 